\title{
Optical absorption and photoelectron collection properties of silicon wafers with conical quantum nanocrystals structure
}

\author{
Yuriy Vashpanov ${ }^{*}$ and Jae-II Jeong
}

A conical form of nano-sized quantum cluster was formed on the surface of p-type crystalline silicon [111] wafer by anode electrochemical etching in HF-based solution. The conical surface is highly effective in absorbing sunlight and transporting photoelectrons to semiconductor material. These are because each cone has a graded band gap with the energy level in the range from 1.1 to 3 $\mathrm{eV}$ which can be considered as consisting of quantum dots in different sizes. Since the boron concentration on the surface of each cone gradually decreases from top to bottom, a continuously varying electrical field is created along the cone height. This electric field is forcing photoelectrons generated in the cone to move rapidly to the direction perpendicular to wafer surface. Hence the drift time of photoelectrons can be less than their recombination time within the thin layer close to the bottom of the cone.

Keywords: Silicon; Nanocrystals; Electrochemical etching; Solar cells

Citation: Yuriy Vashpanov and Jae-II Jeong, "Optical absorption and photoelectron collection properties of silicon wafers with conical quantum nanocrystals structure”, Nano-Micro Lett. 2, 149-153 (2010). doi: 10.5101/nml.v2i2.p149-153

Searching new materials and methods which can increase the efficiency of the solar cell is important in both theoretical and practical application point of view [1]. Now, solar cells with the base of polycrystalline $\mathrm{Cu}(\mathrm{InGa}) \mathrm{Se}_{2}$ (CIGS or CIS with no $\mathrm{Ga}$ ) and of cadmium telluride thin films [2], with triple-junction on the base of GaInP-GaAs-Ge layers [3], with polymer mixtures combined with InP nanowires thin-film [4] are very promising. To further increase the efficiency, silicon solar cells with a tandem structure of silicon quantum dot/crystalline silicon is proposed [5]. However, the solar cells on the crystalline silicon base are currently reached to have efficiency of $24 \%$ [6], and are the most popular technique because there is well-developed production equipment, and the material is cheap and nontoxic.

A method of multilayered semiconductors, where the top layer absorbs short-wave light and the subsequent layers absorb long-wave sun light, is proposed [7]. Semiconductors with band gap energy matching to quantum energy of sun light can be used most effectively for solar cells. However, to cover all solar spectrums, the number of layers should be large. It has shown theoretically that in case of multilayer ( $n=20$ to 25 ) thin film semiconductors with different band gaps in the range from $E_{\mathrm{g} 1}=1.1 \mathrm{eV}$ to $E_{\mathrm{g} 2}=3 \mathrm{eV}$ with a step $\left(E_{\mathrm{g} 2}-E_{\mathrm{g} 1}\right) / n$. It is possible to achieve solar cells with efficiency near $60 \%$ [8]. It is known recently that a semiconductor band gap increases as crystal's geometrical size decreases (quantum nano-size effect) [9]. For example, crystalline silicon quantum dots in different geometrical size from $2 \mathrm{~nm}$ to $6 \mathrm{~nm}$ have a magnitude of band gap energy from 3 to $1.3 \mathrm{eV}$ [10]. Band-gap energy of crystalline silicon is around $1.1 \mathrm{eV}$ [11]. Thus, this idea can be realized today with the help of nanotechnology when a serially connected quantum dots system with size in the range from 1 $\mathrm{nm}$ to $10 \mathrm{~nm}$ or nano-sized cone crystal on a crystalline silicon

Electrical and Computer Engineering Division of Hanyang Institute of Technology, Hanyang University, 17 Haengdang-dong, Seongdong-ku, 133-791, Seoul, South Korea

*Corresponding author. E-mail: vashpanov@hanyang.ac.kr 
surface is fabricated.

In this short communication, a new method of forming conical nanocrystals as anti-reflection layer, on the silicon surface and the preliminary analysis of its light collection efficiency are presented.

A nano-sized crystal having a cone shape has been produced on the surface of p-type crystalline silicon wafer in [111] direction by anode electrochemical etching in HF-based solution. The etching process lasts 5 minutes under the current density around $10 \mathrm{~mA} / \mathrm{cm}^{2}$. Before the etching process, boron is thermally diffused on to the silicon wafer. The boron concentration reaches to $10^{22} \mathrm{~cm}^{-3}$ on the surface of silicon wafer and decreases exponentially as it gets into the inside of the wafer according to Fick's law [12].

The atomic-force microscopy image of the thin layer of nano-sized conic clusters on crystalline silicon wafer, taken by Nanoscope IIIa Dimension 3000TM (Digital Instruments, USA) is shown in Fig. 1. Figure 1 shows that a large number of cones are almost uniformly disposed on the surface. The surface morphology of the silicon wafer is clearly different from that of the porous silicon which is fabricated through the similar electrochemical etching procedure [10]. Apparently, the difference is brought by the thermal diffusion of boron into silicon wafer. The silicon wafer absorbs practically all visible light spectral range, i.e., from violet to near infrared. The measurement shows that it reflects less than $2 \%$ of the sun light in the wavelength range from $300 \mathrm{~nm}$ to $1120 \mathrm{~nm}$. This means that it works almost like a black body, i.e., the cone layer forms an anti-reflection layer on the p-type silicon layer. The small reflectivity values are probably induced by two physical reasons such as evanescing of wave along the surface of the cones and graded band gap semiconductor properties of the cones. It is possible to consider that the coned surface is working as a nanoscale grating (see Fig. 2). In this case, the lights will

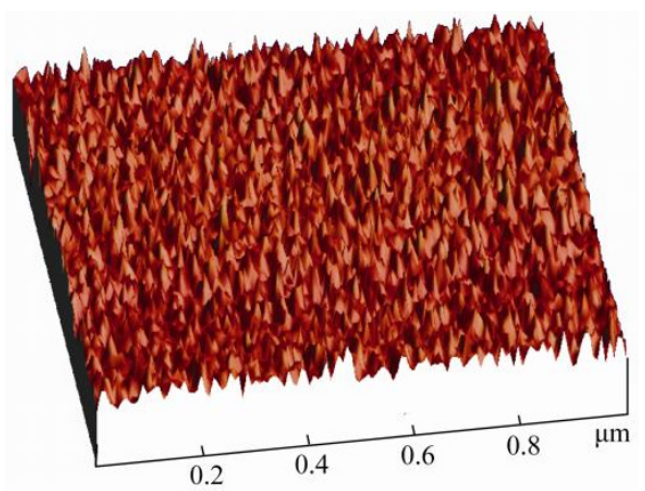

FIG. 1. The atomic-force microscopy image of the thin layer of nano-sized conic clusters on crystalline silicon wafer, taken by Nanoscope IIIa Dimension 3000TM (Digital Instruments, USA) propagate along the coned surface. Hence they are mostly absorbed on the surface of each cone as the evanescent wave does [13]. The graded band gap can only be explained by quantum-size effect, i.e., the cone diameters in nano size increase as getting closer to its bottom. Hence it forms a quantum dot system. This system has a continuously varying band gaps within 1.1 to $3 \mathrm{eV}$. Hence each cone works like a serially connected quantum dot system with different band gap energies. The maximum energy appears at the top of the cones as shown in Fig. 3. The diameter of the top part is

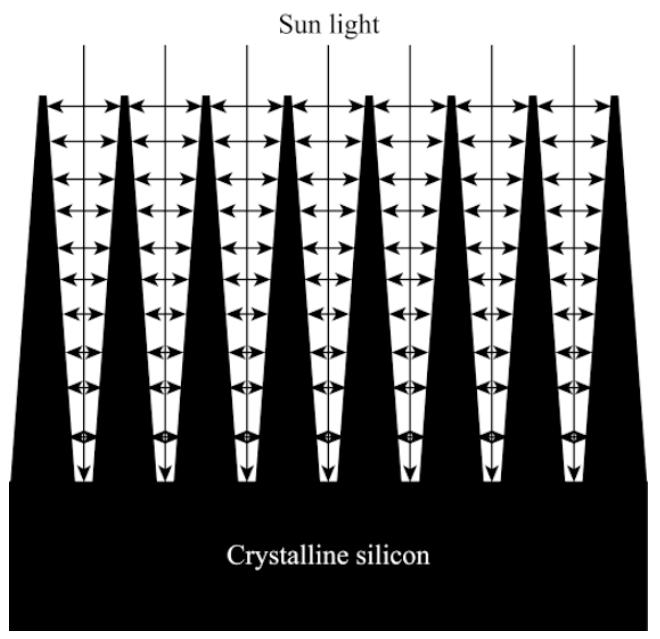

FIG. 2. A model of the coned surface as a nanoscale grating.

approximately $2 \mathrm{~nm}$.

The number of photons from sun light in the waveband within $\lambda_{1}$ to $\lambda_{2}$ that get on square meter of semiconductor surface per second, can be calculated by Plank's radiation law [14]:

$$
\begin{aligned}
N & =\int_{\lambda_{1}}^{\lambda_{2}} N(\lambda, T) d \lambda=\int_{\lambda_{1}}^{\lambda_{2}} \frac{Q(\lambda, T) \times \lambda}{h c} d \lambda, \\
& =\int_{\lambda_{1}}^{\lambda_{2}} \frac{c_{1}}{\lambda^{4}\left[\exp \left(c_{2} / \lambda T\right)-1\right] \times h c} d \lambda
\end{aligned}
$$

Where, $\quad Q(\lambda, T)=\frac{c_{1}}{\lambda^{5}\left[\exp \left(c_{2} / \lambda T\right)-1\right]} \quad$ is the blackbody spectrum (Plank's radiation law),

$c_{1}=2 \pi h c^{2}=3.741832 \times 10^{-16} \mathrm{~W} \cdot \mathrm{m}^{2}$, $c_{2}=h c / k=1.438786 \times 10^{-2} \mathrm{~m} \cdot \mathrm{K}$,

$\lambda$ wavelength of light, $T$ (=5523.3) the black body's temperature in Kelvin of the sun in sea level [15], $h$ is Planck's constant, $c$ is speed of light, and $k$ is Boltzmann's constant [14].

Photons with higher energy are absorbed at the top part of cone. Light with quantum energy less than band gap energy of the cone cannot be absorbed. For example, the quantum of light 


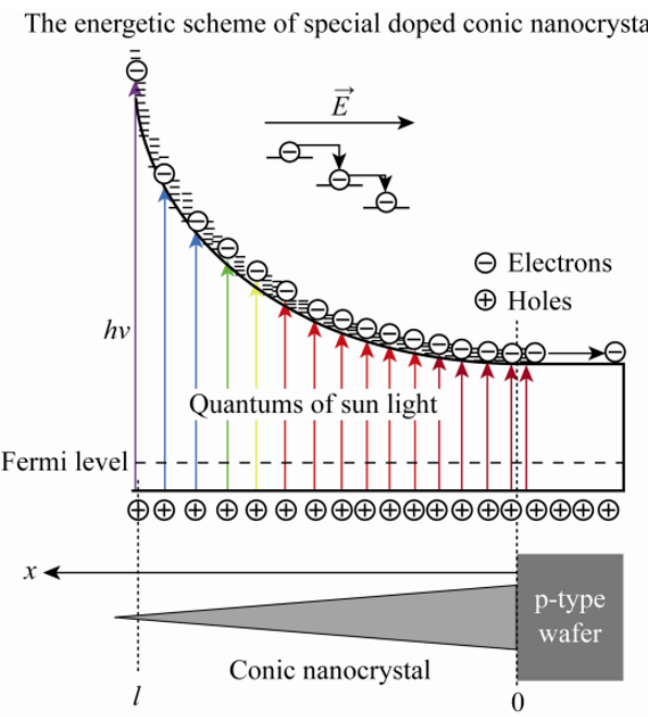

FIG. 3. The configuration of graded band gap structure of conic nanocrystal.

with energy $\sim 1.1 \mathrm{eV}$ cannot be absorbed on the cone surface, because band gap energy of quantum dots are bigger than 1.1 $\mathrm{eV}$. The photons with energy equal to the band gap can be maximally absorbed at the part of the cone, where its diameter corresponds to the band gap. The absorption spectral range of the silicon wafer, $300 \sim 1120 \mathrm{~nm}$ covers much of the solar energy from the sun as shown in the first curve of Fig. 4.

Compared with the silicon wafer with coned layer, silicon crystal absorbs only part of sun light within the waveband 300 $\mathrm{nm}$ to $1120 \mathrm{~nm}$ as shown in curve 2 of Fig. 4. From Eq. 1, the total number of photons absorbed in the silicon crystal for the given $T, N_{\mathrm{c}-\mathrm{Si}}$ can be calculated as

$$
N_{\mathrm{c}-\mathrm{Si}}=\int_{300}^{1120} N(\lambda) F_{a b s}(\lambda) d \lambda,
$$

Where, $F_{a b s}(\lambda)$ is the absorption factor for each wavelength for crystalline silicon [16]. The ratio of $N$ to $N_{\mathrm{c}-\mathrm{Si}}$,

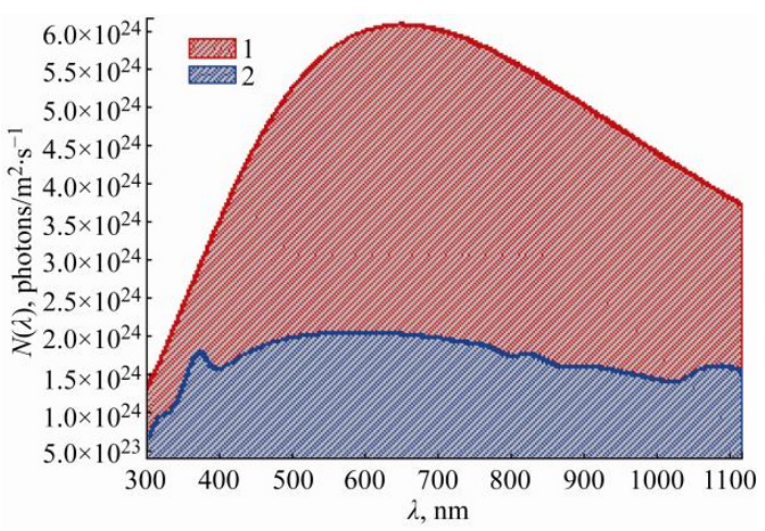

FIG. 4. A photons density per second of solar radiation spectrum (a curve 1) and a absorbing in crystalline silicon photons spectrum (calculated from data of absorption spectrum [16]) (a curve 2). $\gamma_{a b s}\left(\gamma_{a b s}=N / N_{\mathrm{c}-\mathrm{Si}}\right)$ represents the gain achieved by forming coned surface. $\gamma_{a b s}$ is calculated as 2.784 for sample shown in Fig. 1. The efficiency of solar cell increased 2.784 times of that of crystalline silicon, when no other parameter is changed.

Since, the physical reason of efficiency decrease in solar cells is the recombination of photoelectrons in the p-type part and a p-n-junction area of semiconductor solar device [17]. One way of decreasing the recombination rate is to make the drifting time of non-equilibrium photoelectrons from p-type conical clusters shorter than that of the recombination. The graded band gap semiconductors under a special doping profile having a flat valence band as shown in Fig. 3 can create the condition. The electric field formed by the continuously different conductance band gap energies of nano-sized quantum cone crystal as shown in Fig. 3, forces the photoelectrons to move fast in the direction perpendicular to the silicon wafer surface. Thus, the conical clusters are making the photoelectrons to behave like ballistics electrons. These ballistics electrons have a short transit time in p-n- junction area that can lead to reduce recombination losses in the $\mathrm{p}-\mathrm{n}$ - junction.

This exponentially varying doping profile of boron concentration is made before etching. Obviously, this exponential profile is kept even after etching treatment. Thus, the configuration of graded band gap structure as shown in Fig. 3 is achieved. Precise estimation of Fermi level position and band gaps of conic quantum dot systems depend on the boron concentration. The estimation cannot be done easily but it is very important in optimizing technological process of solar cells.

Photoelectrons, which are generated by the trapped photons p-type conical nano-sized clusters, recombine with holes during their transit time period $\tau_{\text {rec }}$ when no electric field exists. This recombination will lead to efficiency loss for solar cells. However, with electrical field in conical clusters, the photoelectrons transit the p-type layer shorter than $\tau_{r e c}$. If $\tau_{\text {drift }}$ the transit time under the electric field is considerably less than $\tau_{\text {rec }}$, the current losses in anti-reflection layer will be reduced. Determining the transit time in nanostructures is a really complex problem. However, the drift time from the semiconductors physics, which is based on quantum mechanics laws is used, the effective electric field is calculated as $\bar{E}=\frac{\Delta E_{C}}{e \cdot l}$ for the profile of conductance band as shown in Fig. 3, where $\Delta E_{C}$ is gradient of conducting band gap energy, $e$ is electron charge and $l$ is the height of conical clusters. In this 
case, $\tau_{d r i f t}$ is calculated as $\tau_{d r i f t}=\frac{l}{\mu_{n} \bar{E}}=\frac{l^{2}}{\mu_{n} \Delta E_{c}}$. The condition $\tau_{\text {drift }} \leq \tau_{\text {rec }}$ will be achieved when $l \leq \sqrt{\mu_{n} \Delta E_{c} \tau_{\text {rec }}}$.

For silicon, since $\mu_{n} \approx 1400 \mathrm{~cm}^{2} / \mathrm{Vsec}, \tau_{\text {rec }}=10^{-12}$ sec [11] and $\Delta E_{c}=0.1 \div 2.9 \mathrm{eV}$, the maximum $l$ value satisfying the relationship is around $118 \mathrm{~nm}$. Thus, photoelectrons can go through the p-type anti-reflected layer without significant recombination when the cone height is less than $100 \mathrm{~nm}$. In practice, this condition can be easily obtained under the current technology of manufacturing solar cells. Since it is desired to decrease the height of each cone for the minimization of internal resistance of the conical cluster, photoelectrons will leave more rapidly the p-type layer without recombining with holes. The minimum speed of the photoelectrons can be around $10^{6} \mathrm{~m} / \mathrm{s}$. This speed is more than 4 times of that of thermal electrons at room temperature in the silicon crystal. The speed of thermal electrons is around $2.3 \cdot 10^{5} \mathrm{~m} / \mathrm{s}$ [11]. For this reason, the efficiency of solar cell will be increased compared with that of the solar cells based on crystalline silicon by reducing the recombination rate 4 times.

It is considered that the current losses in the solar cells based on crystalline silicon are attributed to different physical effects: The thermalization of photoelectrons causes $29.2 \%$ loss, the incomplete collection of photoelectrons $4.5 \%$, added shunting resistor $4.7 \%$, and the recombination in junction area $19.2 \%$ [17]. With the anti-reflection layer, the losses due to the photoelectron thermalization and the incomplete collecting of photoelectrons will be eliminated by the graded band gap presence. Ballistics electrons have a higher probability of passing the cones. Therefore the efficiency of solar cell with conical nano-sized cluster layer can be characterized with the photocurrent $I_{p h}$, the recombination current $I_{r e c}$, and current through added shunting resistor, $I_{f}$. The efficiency increment $\gamma$ can be calculated as

$$
\begin{aligned}
\gamma & =\frac{\gamma_{a b s} I_{p h}-\gamma_{b} I_{r e c}-I_{f}}{I_{p h}-I_{r e c}-I_{f}}=\frac{\gamma_{a b s} I_{p h}-\gamma_{b} \xi_{r e c} I_{p h}-\xi_{f} I_{p h}}{I_{p h}-\xi_{r e c} I_{p h}-\xi_{f} I_{p h}} \\
& =\frac{\gamma_{a b s}-\gamma_{b} \xi_{r e c}-\xi_{f}}{1-\xi_{r e c}-\xi_{f}}=3.539
\end{aligned}
$$

Where $\gamma_{b}, \xi_{\text {rec }}$ and $\xi_{f}$ represent transit time ratio of ballistic electrons to thermal electrons, and losses by recombination and shunting resistor, respectively. The efficiency of commercial silicon solar cell is about 15 to $20 \%$ [18]. With the assumption of preserving other parameters of solar cells, the efficiency should increase to $\gamma$ times, i.e., the efficiency will be calculated as $3.539 \times(15 \sim 20 \%)=53 \sim 70 \%$.

As the conclusion, generating ballistic electrons with the conical form quantum clusters layer will lead to a significant improvement in the solar cell efficiency based on crystalline silicon. The anti-reflecting and photoelectrons collecting thin layer can easily be formed by the etching method described before. Silicon solar technologies will have good perspectives in competing with other materials of high efficiency. The conical form quantum clusters layer will not essentially increase the manufacturing cost of solar cells. For more impactful and functional than silicon, it may be necessary to use a germanium (or another narrow-band-gap semiconductor), which has the band gap energy of $0.7 \mathrm{eV}$. In this case, more infrared spectral range of the sun light will be absorbed. This means that the efficiency will be increased more.

Authors are grateful to professors Jung-Young Son and Kae-Dal Kwack for useful discussion and remarks. This study was supported by Hanyang University's Brain Korea 21 program.

\section{Received 19 April 2010; accepted 9 July 2010; published online 27} July 2010.

\section{References}

1. M. McGehee Solar Cells in 2009 and beyond, (KAUST, 2009).

2. MRS BULLETIN, Harnessing Mater. Energy 33, 355 (2008).

3. R. R. King, D. C. Law, K. M. Edmondson, C.M. Fetzer, G. S. Kinsey, H. Yoon, R. A. Sherif and N. H. Karam, Appl. Phys. Lett. 90, 183516 (2007). doi:10.1063/1.2734507.

4. C. J. Novotny, E. T. Yu and P. K. Yu, Nanolett. 8, 775 (2008). doi:10.1021/n1072372c.

5. Eun-Chel Cho, Sangwook Park, Xiaojing Hao, Dengyuan Song, Gavin Conibeer, Sang-Cheol Park and M. A. Green, Nanotechnology 19, 245201 (2008). doi:10.1088/0957$\underline{4484 / 19 / 24 / 245201 .}$.

6. Forum for solar cells: http://www.solarserver.de/.

7. G. Raushenbakh, The directory on designing of solar batteries, (in Russian, Moskow, Energoizdat, 1983).

8. V. V. Serdyuk, Physics of solar cells, (in Russian, Logos, Odessa, 1994). 
9. N. Daldosso and L. Pavesi, Laser \& Photon. Rev. 3, No. 6, 508 (2009). doi:10.1002/lpor.200810045.

10. Bisi, S. Ossicini and L. Pavesi, Surf. Sci. Rep. 38, (2000).

11. Kwok K. Ng Complete guide to semiconductor devices, (McGraw Inc., International edition, 1995).

12. S. W. Jones Diffusion in silicon, (IC Knowledge LLC, 2000).

13. Nanophotonics Device Group: http://www.leakymodereso nance.com.

14. W. G. Driscoll and W. Vaughan, Handbook of Optics, (MCGRAW-HILL Company, 1978).
15. Solar Radiation Hand Book (MNRE, Indian Metrological Department, 2008).

16. J. R. Chelikowsky and M. L. Cohen, Phys. Rev. B 14, 2 556 (1976).

17. A. L. Fahrenbruch and R. H. Bube, Fundamental of solar cells. Photovoltaic solar energy conversion, (New York, 1983).

18. M. A. Green, Physica E 14, 65 (2002). doi:10.1016/ $\underline{\text { S1386-9477(02)00361-2. }}$. 